\title{
OBITUARIO
}

\section{Lobo Orensanz (1945-2015) y su contribución al conocimiento de los poliquetos (Annelida)}

\author{
Lobo Orensanz (1945-2015) and his contribution to the study of polychaetes (Annelida)
}

\author{
María Emilia Diez ${ }^{1}$, Alejandro Tablado², Fabrizio Scarabino ${ }^{3}$, \\ Juancho Orensanz $4^{4}$, Luis F. Carrera-Parra ${ }^{5}$, Rodolfo Elías ${ }^{6}$ \\ y Sergio I. Salazar-Vallejo ${ }^{5^{*}}$
}

\begin{abstract}
${ }^{1}$ Centro Nacional Patagónico, Bv. Almirante Brown 2915, Puerto Madryn, Argentina
${ }^{2}$ Museo Argentino de Ciencias Naturales Bernardino Rivadavia, Acceso A Ángel Gallardo 490, Buenos Aires, Argentina ${ }^{3}$ Museo Nacional de Historia Natural y Dirección Nacional de Recursos Acuáticos, 25 de mayo 582, Montevideo, Uruguay ${ }^{4}$ Balcón de Los Santos, Avenida Costanera Orensanz 516, Santa Clara del Mar, Argentina

${ }^{5}$ El Colegio de la Frontera Sur, Ave. Centenario km 5.5, Chetumal, México.* savs551216@hotmail.com, ssalazar@ecosur.mx ${ }^{6}$ Universidad Nacional de Mar del Plata, Dean Funes 3350, Mar del Plata, Argentina
\end{abstract}

\begin{abstract}
José María Orensanz, better known as 'Lobo' since his childhood, made different scientific contributions on the taxonomy of marine or estuarine annelids, as well as in ecology and artisanal fisheries of several benthic invertebrate groups. Lobo's contributions in fisheries were described elsewhere; in this eulogy, we make a brief review about some salient features of his life and about his efforts in the study of polychaetes. In particular, Lobo's 37 publications on benthic ecology or poychaete taxonomy are detailed, and in those dealing with polychaetes he dealt with 18 mostly benthic families and made 3 papers on pelagic species; he proposed 9 genera and described 29 species. Three annexes are included listing his publications, the taxa he proposed or described, and the taxa named after him including a genus and 18 species.
\end{abstract}

Key words: Argentina, Patagonia, Brazil, Uruguay, taxonomy

\begin{abstract}
Resumen.- José María Orensanz, conocido como 'Lobo' desde niño, realizó investigaciones en sistemática de anélidos marinos o estuarinos y en la ecología y pesquerías de varios grupos de invertebrados bénticos. Se han reseñado sus contribuciones sobre el uso de los recursos pesqueros en otra parte; en este obituario presentamos una breve reseña sobre algunos aspectos de su vida y sobre sus afanes en el estudio de los poliquetos. En particular, se enlistan las 37 contribuciones en ecología del bentos y sobre las relativas a la sistemática de los anélidos poliquetos, en donde comprendió 18 familias de bénticos e hizo varios trabajos sobre pelágicos; en ellos, propuso 9 géneros y describió 29 especies. Se acompaña el documento con 3 anexos con las listas de sus publicaciones, los taxa propuestos o descritos, así como los taxa nombrados en su honor por varios especialistas que incluyen un género y 18 especies.
\end{abstract}

Palabras clave: Argentina, Patagonia, Brasil, Uruguay, taxonomía

\section{INTRODUCCIÓN}

Hacer una semblanza sobre José María Orensanz, mejor conocido como 'Lobo', es una tarea ingrata porque pudiendo ser un homenaje más que merecido en vida, deviene obituario por su repentina y dolorosa partida a principios del 2015. 'Lobo' era un investigador prodigioso porque tenía un conocimiento enciclopédico de la fauna de invertebrados del Atlántico sudoccidental, y no sólo de los bénticos, lo que conjugaba con dominio de aspectos teóricos y prácticos en laboratorio y campo relevantes en los terrenos de la zoología y biogeografía marinas. Las preguntas, la formación y capacidad para responderlas adecuadamente eran igual de sorprendentes y, sobre todo, pioneras en campos de ecología, biogeografía marina, biología pesquera y evolución de los grupos que le interesaban tales como poliquetos, crustáceos y moluscos. Todo esto basado en un balance asertivo del conocimiento y del desconocimiento taxonómico y faunístico del grupo en cuestión, asunto en que era líder al generar conocimiento y en promover su generación. Del mismo modo, manejaba sin dificultad herramientas cuantitativas variadas, incluyendo programación informática desde los años 80 .

Su partida ha sido prematura y tan inesperada como dolorosa; deja un vacío afectivo y académico que no tienen reemplazo. Es posible definir a 'Lobo' con tres 
palabras: humildad (nunca se subió a ningún pedestal), generosidad (en brindar su saber y sus cosas) y respeto (por el prójimo y sus conocimientos). Estas consideraciones no son originales ya que otros ya han destacado estas enormes cualidades de su entrañable personalidad.

Sus actividades como biólogo pesquero han sido reconocidas en otras ocasiones y foros; además, su generosidad, laboriosidad y amplitud de miras han sido reseñadas más o menos detalladamente ${ }^{1}$. Además, la Universidad de Washington estableció un fideicomiso para impulsar los estudios de posgrado para jóvenes latino-americanos en esas temáticas ${ }^{2}$.

Debe resaltarse que su generosidad incluía abrir las puertas a sus estudiantes e investigadores a un ambiente de armonía y de respeto mutuo, incluyendo el brindar alojamiento a investigadores y estudiantes en un anexo de su casa, mantenida para tales fines, conocida como 'El Mostro' o 'TheMonster' por las inusuales dimensiones de ese edificio. Era muy generoso con sus estudios e ideas, sugiriendo nuevas pautas o líneas de investigación; su visión sabia hacía posible lo 'imposible', tanto en la investigación ensamblando temas y disciplinas, como en las relaciones humanas reuniendo personas con diferentes formas de trabajo o de pensar. 'Lobo' lograba encontrar un punto común con atinada predisposición. Ha dejado muchas enseñanzas y es parte importante de nuestras vidas.

\section{INFANCIA}

'Lobo' nació en la primavera austral, en Mar del Plata, Argentina, un 9 de octubre de 1945. Sus padres fueron José María Orensanz y María Luisa Barreiro, ambos descendientes de inmigrantes españoles; los paternos de Aragón, los maternos de Galicia. Su infancia transcurrió en el seno de una familia muy unida y con cierta holgura económica (hay algunos detalles de reuniones familiares disponibles en línea $)^{3}$.

Su tío Antonio tenía visión inmobiliaria e impulsó, junto con el padre de 'Lobo', la fundación de Santa Clara del Mar en 1949, a unos 18 km de Mar del Plata. Don Antonio tenía el antecedente de impulsar la fundación en 1948 de Santa Elena, otra población costera ubicada a unos $2 \mathrm{~km}$ de Santa Clara.
El padre de 'Lobo' había hecho carrera en el teatro. Discípulo de Galina Tolmacheva (Dubatti 2009), fundó el Teatro-Escuela ABC en Mar del Plata (Santa Clara del Mar) e invitó a su maestra para que fuera directora, cargo en el que duró 2 años (1955-1956). Posteriormente, fundaría un teatro escuela en Santa Clara del Mar, lo que remarca su interés por las artes. La puesta en escena de Los Justos de Albert Camus, asociado con la relación con la escuela de Teatro de la Universidad Nacional Eva Perón, desencadenaron malestar en el Estado y motivó su persecución y encarcelamiento. Debieron haber sido tiempos difíciles para toda la familia.

La escuela primaria o elemental la realizó en Santa Clara del Mar, la secundaria en varias ciudades porque la familia cambiaba residencia durante el invierno; se mudó a Buenos Aires para el resto de su educación. Vivió en el departamento de los abuelos maternos, lo que implicaba viajar diariamente en tren los $60 \mathrm{~km}$ que median entre Buenos Aires y La Plata. Fue en la Facultad de Ciencias Naturales y Museo de la Universidad Nacional de La Plata, en dónde obtuvo el doctorado en 1986 con su estudio monográfico sobre los eunicemorfos de mares antárticos y subantárticos.

De los intereses por los organismos en general y de sus actividades como científico en formación, Patricia Sánchez de Bustamante compartió uno de sus recuerdos generados en 1955: El 'Lobo' tiene 10 años y yo 8. Me doy cuenta que compartimos una silenciosa timidez bajo preguntas y respuestas, pero también el embeleso por la magia de las mariposas. Vamos recorriendo los hermosos ejemplares de diversas formaciones de diseños, colores y reflejos, que el 'Lobo' ha puesto en hileras prolijamente ensartadas con un alfiler. ¿No sufren Lobito? Se me esfuma la respuesta pero quedó nítida su sonrisa transformándose en crisálida. Para mis ojos de niña, ese chico de pelo negro revuelto, camisa blanca, hermoso, flotaba en ese espacio de semi-penumbra atravesado por hilos tenues de luz marina con motitas de polvo. En ese su mundo que me compartía, de sueños, de aventura apasionada por insectos poblados de enigmas para mi cabecita soñadora, creo que una parte de mi quedó atrapada como otra pequeña mariposa, en éxtasis.

\footnotetext{
${ }^{1}<$ http://claustronodocenteunpsjb.blogspot.mx/2015/01/el-lobo-jose-maria-orensanz.html>

${ }^{2}<$ https://safsfishline.wordpress.com/2015/01/09/establishment-of-the-lobo-orensanz-endowed-fund-for-student-support>

${ }^{3}<w w w . y o u t u b e . c o m / w a t c h ? v=1 z z \_V 6 f a s 5 c \& l i s t=U U \_6 A 8 d 5 V W P-y t X f P h o z r g f w \&$ index $=23>$
} 
En cuanto a la génesis de los intereses de 'Lobo' por los organismos marinos se sabe poco o nada, aunque viviendo en el litoral, habría variedad de organismos en los arribazones o sólo haría falta esperar la bajamar. Una de sus fotos de su temprana adolescencia muestra su gusto e interés por andar en la playa en compañía de Esteban Garvie (Fig. 1), quién recuerda que su casa 'poseía un gran ventanal por donde se contemplaba tanto inmensidad marítima como la pampeana y que divisaba con curiosidad el paso ocasional de un muchachito apenas un poco mayor que yo. Munido generalmente de un palo al modo de bastón de caminante, con botas de goma, y a veces vistiendo un sombrero algo extravagante, se dirigía a la costa y descendía a la playa'. En donde, 'Lobo encontraba presencias fascinantes. Innumerables caracoles de miniatura, piedritas de origen intrigante, pequeños moluscos en distintas fases de su ciclo vital, diminutos cangrejos que a veces simulaban quietud, o huían ante nuestra curiosidad. Algas de variada morfología. Huevos de raya, tiburón o de caracol, y miríadas de pequeños objetos cuyo origen daba lugar a todo tipo de especulaciones'.
Esteban no está seguro de la fecha en la que descubriría los anélidos marinos pero recuerda con viveza que en uno de esos paseos 'Lobo toma una piedra de gran tamaño, y la arroja contra una formación de tosca en aquella zona que se había convertido en su observatorio privilegiado. La arenisca se desprende en fragmentos y allí, en el surco creado por el golpe, se retorcía el largo cuerpo casi translúcido de una lombriz de mar. 'Lobo' lo recoge y lo deposita en un frasco preparado con agua y pasó a relatar la historia de aquel bicho, cuya existencia pasa ignorada para los paseantes playeros'.

\section{VIDA PROFESIONAL}

'Lobo' comentó que su interés en los poliquetos era consecuencia del impacto del Dr. Rioja. Mencionó que antes de la universidad no disfrutaba mucho la escuela por lo que prefería asistir al Instituto de Biología Marina en Mar del Plata, en donde le dejaban estar en los laboratorios y usar los microscopios para examinar los organismos que encontraba y en donde también contribuía con valiosas muestras como los sipuncúlidos que fueron aportados a Analía Amor (Amor 1964).

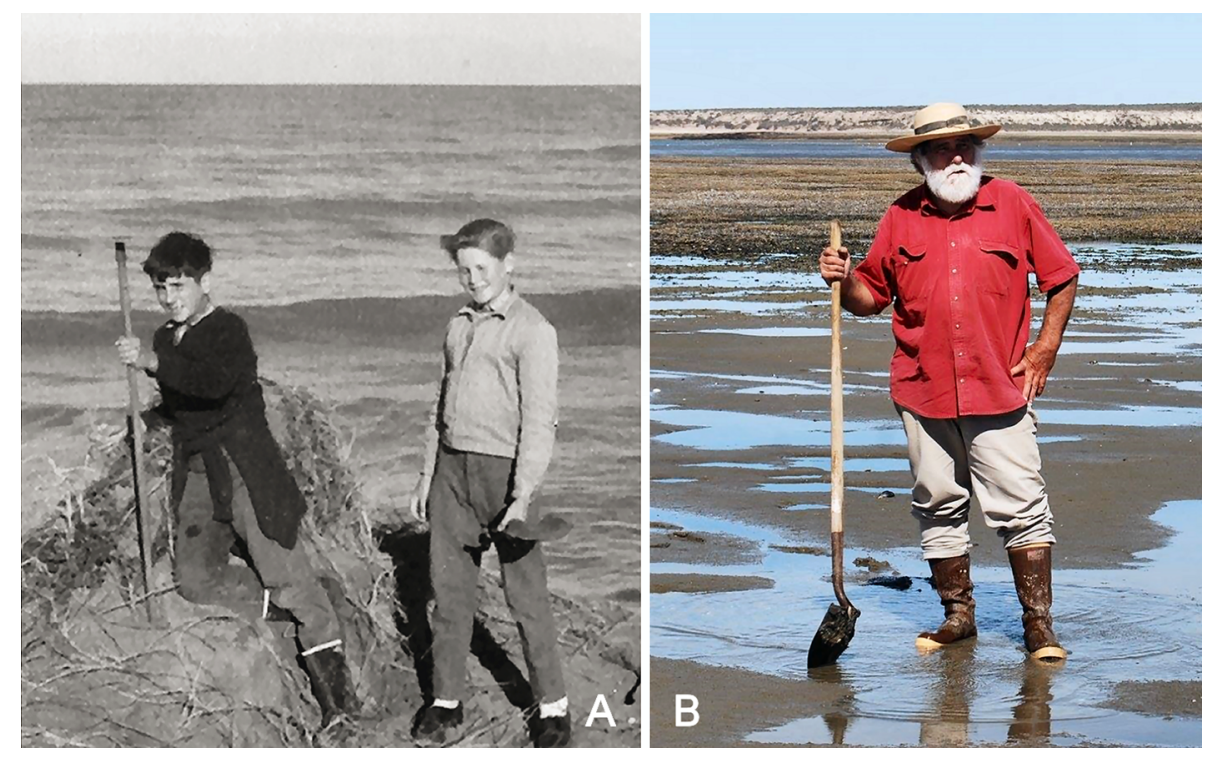

Figura 1. Siempre curioseando y explorando; siempre bien equipado. A) Lobo y Esteban Garvie en la playa de Santa Clara del Mar en 1956 (tomado de Orensanz 2010); B) Lobo paleando en Riacho, en la base del Golfo de San José, en una bajamar veraniega del 2010. Autores de la foto: María Luisa Barreiro (A) y Vasily Radashevsky (B) / Always curious and exploring, always well-suited. A) Lobo and Esteban Garvie in the Santa Clara del Mar beach in 1956 (from Orensanz 2010); B) Lobo shoveling in the Riacho beach, Golfo de San José, in a 2010 summer low tide. Photo credits: María Luisa Barreiro (A) and Vasily Radashevsky (B) 
En una ocasión durante 1962, Rioja que estaba en un seminario sobre biogeografía de organismos bénticos (Caso 1962), entró al laboratorio y al notar que 'Lobo' examinaba unos poliquetos, comentó que le concedió 'el curso más intensivo y motivador sobre poliquetos que uno podría imaginar'. 'Lobo' lo recordó así (Orensanz 2009): 'En aquel entonces yo tenía 16 años y era estudiante de secundaria, ya apasionado por la historia natural. Durante la semana del simposio abandoné la escuela para asistir a la reunión - fui el único espectador externo. Correr el riesgo tuvo su premio: aquel hombre sabio y venerado se acercó a conversar conmigo, escuincle fuera de contexto entre tantos académicos notables'.

Entonces, parece que el interés por los poliquetos surgió antes de empezar la carrera. Sin embargo, es posible que fuera durante esta etapa que consiguió cierta comprensión o dominio del latín y del alemán que luego le serían de gran ayuda.

'Lobo' hizo el servicio militar en una localidad remota de la Patagonia (más remota en la década de los 1960). Allí colectó durante meses material zoológico con destino al Museo de La Plata, y en la Universidad hizo su licenciatura en Zoología. Recordaba particularmente material de ortópteros que fue incluido en publicaciones del entomólogo Ronderos y ¡las dificultades para tener un calzado de su talla que le permitiera sobrellevar el frío!

Santiago Raúl Olivier fue quien apoyó mucho del trabajo de 'Lobo' antes de su exilio. Por diferencias con el zoólogo argentino Raúl Ringuelet, no se le permitió a 'Lobo' tener a Olivier como orientador para un doctorado, aduciendo que este último era ecólogo y no taxónomo. En realidad, Olivier tenía antecedentes incuestionables como taxónomo luego de la publicación de una extensa monografía sobre cladóceros argentinos (Olivier 1962). Con la recuperación de la democracia en Argentina, 'Lobo' pudo concretar un doctorado en la UNLP, presentando una tesis sobre Eunicemorpha, publicada en buena parte en su monografía sobre el grupo (Orensanz 1990). Para ello, la Dra. Zulma Ageitos de Castellanos (malacóloga) avaló académicamente la tesis doctoral.

En los 1970 Santa Clara del Mar no era más que unas cuantas casas donde los Orensanz no sólo tenían una sino que eran dueños de la hostería del lugar, La Posta del Angel, que ocupaba toda una manzana (hectárea) del ejido urbano. En este sitio 'Lobo' guardó durante sus años de exilio en USA mucho del material de poliquetos que no pudo llevarse. Algo de ese material (principalmente lo ya publicado) lo depositó en el Museo Argentino de
Ciencias Naturales 'Bernardino Rivadavia' antes de su partida y a su regreso volvió a depositar otra tanda cuando tuvo el tiempo para desempolvar lo que había guardado y protegido en la propiedad familiar.

Durante sus estudios de Licenciatura en Zoología en la Universidad de La Plata había mucho énfasis en Sistemática y Nomenclatura, con lo que era obligatorio consultar bibliografía original. En esas búsquedas, era evidente que muchas de las descripciones de especies habían sido hechas bajo condiciones difíciles e incluso durante las guerras. Estas condiciones le orillaron a reflexionar sobre la interfase investigador-sociedad, respecto a la responsabilidad del ejercicio profesional.

En realidad, durante los años oscuros de la Argentina, 'Lobo' no fue perseguido directamente ya que no militaba políticamente, pero fue perseguido ideológicamente o directamente acosado. Al igual que a muchos otros investigadores del entonces Instituto de Biología Marina de Mar del Plata, le fue prohibida la entrada a dicho instituto debido a 'purgas', mismas que fueron producto de 'señalamientos' hacia personas demasiado progresistas, o que por su brillante desempeño parecían competencia desleal en un panorama de falta de entusiasmo o mediocridad.

Por dichos problemas, 'Lobo' generó el 'Laboratorio de Comunidades Bentónicas, Gabinete Abierto - Santa Clara del Mar' en una propiedad familiar en Santa Clara del Mar, para lo que generó papel membretado y sobres oficiales (Fig. 2). Allí generó el catálogo de poliquetos de la provincia biogeográfica magallánica (mimeografiado) y apoyó junto a su entonces compañera, Anamaría Escofet (Mona) a investigadores más jóvenes en igual situación que ellos. Esto fue un desplante de rebeldía pacífica y creativa que devino relevante para sus intereses de investigación. Poco después, decidieron probar suerte en el CENPAT, en donde una funcionaria debía responder periódicamente ante las autoridades para testificar el comportamiento 'no subversivo' de 'Lobo' y 'Mona'. En este contexto autoritario y represivo, ambos deciden emigrar primero a México y luego a USA en donde fueron a la Universidad de Washington en Seattle, porque un colega, Martin Hall, ya había llegado y lo recomendaba mucho.

La serie de estudios sobre poliquetos de la provincia biogeográfica argentina se basó en materiales que tenían colegas en el mismo instituto, así como otros disponibles en los museos de varias ciudades de la región. Para la generación de dicha serie 'Lobo' mantuvo una estructura 


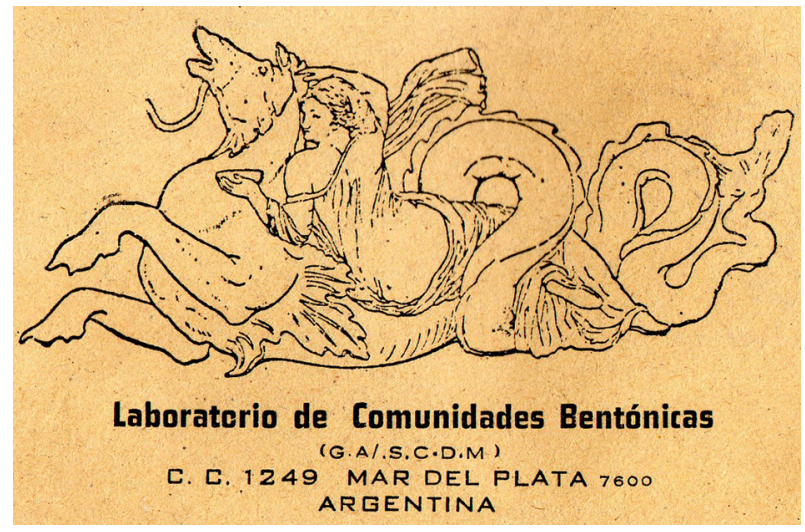

Figura 2. Logo en los documentos oficiales del Laboratorio de Comunidades Bentónicas. Representa a Doris, madre de Amphitrite, que lleva un regalo de bodas montada sobre un hipocampo. Lobo la calcó del tomo de $\mathbf{1 8 9 0}$ de 'La llustración Artística' (Barcelona; 1882-1916). (Cortesía: Juancho Orensanz; ver <http:// en.wikipedia.org/wiki//Altar_of_Domitius Ahenobarbus $>$ ) / Logo from the official documents of the Laboratorio de Comunidades Bentónicas. It shows Doris, mother of Amphitrite, carrying a wedding present mounted over a seahorse. Lobo copied it from the 1890 volume of 'La llustración Artística' (Barcelona: 1882-1916) (courtesy: Juancho Orensanz; see: <http://en.wikipedia.org/wiki/Altar_of_Domitius_Ahenobarbus>)

híbrida combinando la organización en serie que había hecho Enrique Rioja, que eran esencialmente faunísticos con buenas ilustraciones, claves de identificación y dominio de la literatura, con los de Olga Hartman que combinaban la faunística con evaluaciones críticas sobre la composición de las familias, de las sinonimias entre géneros, o sobre la delimitación de especies. Debe mencionarse que Hartman y Rioja pensaban que había buen número de especies cosmopolitas o de amplia distribución, al tiempo que eran capaces de reconocer patrones distintos y proponerlos como géneros independientes o describirlos como nuevas especies.

No obstante, dado que los poliquetos son abundantes y variados lo que representa siempre una mayor cantidad de trabajo de identificación si se comparan con grupos mejor conocidos o menos abundantes, los análisis no podían ser muy detallados para todas las especies, por lo que una organización graduada en contribuciones por familia o al agrupar algunas familias más o menos asociadas, permitiría mejor calidad en la interpretación. Con este enfoque, 'Lobo' consiguió comprender la literatura original y reinterpretar las delimitaciones morfológicas de géneros y especies, de modo que propuso el restablecimiento de varios taxa, algo que era contrario a la opinión de otros especialistas. 'Lobo' reconoció repetidamente la asesoría y respaldo de Santiago Raúl Olivier; sin embargo, tuvo que conocer de manera regular el latín y el alemán para poder comprender las publicaciones en esos idiomas o las descripciones que se estilaban en el primero durante muchos años.

En 2009 'Lobo' participó en su primera reunión de poliquetos durante el II Simposio Latinoamericano de Poliquetos en Mar del Plata. En su presentación 'Estado actual del conocimiento de los poliquetos en Argentina' incluyó una reseña personal sobre su desarrollo y un mapa del sitio donde realizó algunos de sus descubrimientos en poliquetos, justo frente a su casa en Santa Clara del Mar. Era su anhelo que alguien continuara muestreando allí. Grande fue nuestra sorpresa cuando Rodolfo Elías le comentó que sus estudios de impacto ambiental en la descarga de aguas servidas de Mar del Plata habían tomado ese mismísimo sitio de referencia. 'Lobo' invitó a los interesados y permitió atravesar su propiedad para acceder al sitio, lo que ahorró una enorme distancia a pie y el cansancio por volver cargados de muestras.

Una de las cualidades interesantes sobre 'Lobo' es que mecanografiaba usando sólo el índice derecho (Fig. 3 ), con ayuda ocasional del índice izquierdo para tornar a mayúsculas. Le gustaba decir que 'todo lo que he escrito, lo hice con este dedo' y al mostrar el dedo orgulloso lo enfatizaba más, mientras que Ana Parma terciaba: 'es increíble lo rápido que escribe'.

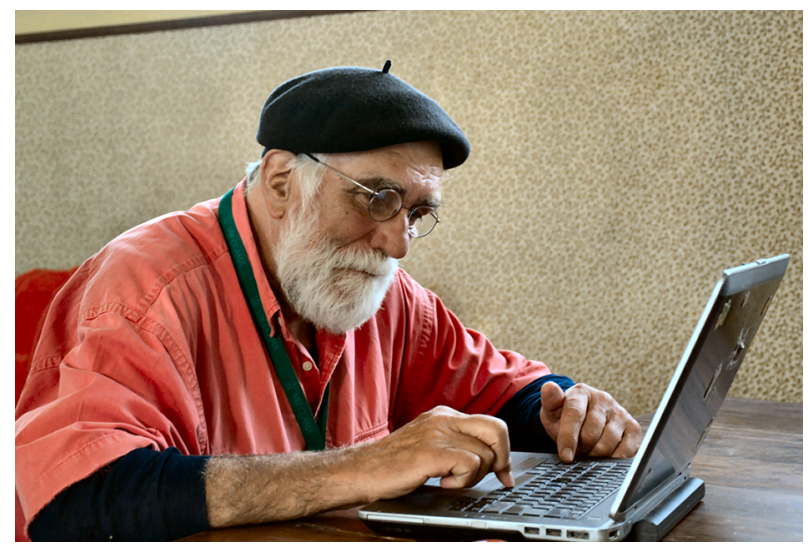

Figura 3. Lobo mecanografiando con el índice derecho (autor de la foto: Daniel Ruzzante / Lobo typewriting with a single finger (photo credit: Daniel Ruzzante) 


\section{ESTUdiantes y Futuro}

El gran éxito y repercusión que tuvo 'Lobo' en la biología pesquera contrasta con lo realizado en el estudio de los poliquetos. Cuando se le preguntaba sobre la razón para no impulsar a los jóvenes al estudio de ese tema, contestaba que le parecía que no habría opciones de empleo, de modo que prefería evitarlo. Sin embargo, hay varios estudiantes que fueron lo suficientemente tenaces como para buscar una vida profesional en el estudio de los poliquetos y que consiguieron orientación de parte de 'Lobo': Agustina Ferrando, Berenice Trovant y María Emilia Diez. De las tres, parece que es sólo la última la que mantiene el interés. En México, su generosidad para compartir experiencias e incluso avances concretos, cristalizó en dos de nosotros al participar en los comités tutelares correspondientes (lfcp, sisv).

Además, directa o indirectamente, Claudia Bremec y Rodolfo Elías son también sus hijos académicos. Los primeros pasos de estos investigadores como becarios, aparentemente alejados de buena supervisión, fueron apoyados por correspondencia con el 'Lobo'.

La obra de 'Lobo' en el estudio de los poliquetos de la región biogeográfica argentina y de la Antártida es tan copiosa que podría dar la impresión de que no hay mucho por hacer (Anexo 1). Todo lo contrario. Dejó una serie de manuscritos pendientes, muchas ilustraciones y comentarios sobre especies que ameritan un esfuerzo adicional para seguir clarificando la composición faunística regional. Estamos seguros que a pesar de su resistencia, 'Lobo' también deseaba una mejora general de la ciencia en Argentina y en particular en el estudio de los anélidos poliquetos, un grupo que a pesar de sus múltiples ocupaciones en otros terrenos, nunca dejó de atender en cuanto había oportunidad.

\section{Publicaciones y nuevos taxa}

'Lobo' inició como ecólogo de comunidades bénticas en una publicación colectiva (Olivier et al. 1966) y se mantuvo en esa misma dirección en otras 2 publicaciones (Olivier et al. 1972a, b). Sin embargo, se abocó con mayor profundidad al estudio de los poliquetos en una serie de contribuciones de mayor alcance geográfico al considerar la provincia biogeográfica argentina. Estos esfuerzos fueron de gran intensidad porque en unos 10 años realizó una impresionante serie de contribuciones.

Antes de empezar a publicar los documentos que serían parte de la serie, participó en una revisión de los poliquetos de aguas salobres de Buenos Aires (Orensanz
\& Estivariz 1972), otros 2 sobre poliquetos pelágicos, uno sobre el Atlántico sudoccidental (Orensanz \& Ramírez 1973) y encabezó otro más sobre la Antártida (Orensanz et al. 1974), más un cuarto sobre la faunística de los poliquetos de Río Grande do Sul (Orensanz \& Gianuca 1974).

Para la serie mencionada inició con los poliquetos errantes y consideró los afrodítidos y redefinió Aphroditella Roule, 1898 (Orensanz 1973b). En su trabajo sobre los crisopetálidos, analizó la delimitación de los géneros y presentó una clave para separarlos (Orensanz 1973a), pasando luego a los mandibulados como los dorviléidos (Orensanz 1974a), en los que propuso un género nuevo. En ese mismo año, en su trabajo sobre los lumbrinéridos (Orensanz 1974b) 'Lobo' transformó la taxonomía de la familia ya que restableció Augeneria Monro, 1930 y propuso 2 géneros nuevos. Siguieron luego los onúfidos (Orensanz 1974c), para terminar con los oenónidos (Orensanz 1974d). Entonces siguió con algunas familias como los poligórdidos (Orensanz 1975b), acrocírridos (Orensanz 1975c) en el que redefinió la familia y cuya organización fue ratificada (Salazar-Vallejo et al. 2007), y terminó la serie con los poeciloquétidos y cosúridos (Orensanz 1976a).

Esta producción extraordinaria fue acompañada de otras publicaciones relevantes sobre la fauna regional tales como una compilación de las especies de la región magallánica (Orensanz 1976b), así como 3 contribuciones para libros sobre biota acuática (dulceacuícola) del continente americano (Orensanz 1977, 1981, 1982). 'Lobo' volvería a ser autor único con su monografía de los eunicemorfos de los mares antárticos y subantárticos (Orensanz 1990), que fue sin duda su obra más ambiciosa y acabada, por lo que merece una consideración detallada.

En esa gran obra, además de la caracterización de las especies de la región, se describieron 9 nuevas especies, se propusieron 4 nuevos géneros y otro fue restablecido, e hizo consideraciones biogeográficas sobre el grupo. El trabajo ha sido muy influyente para el estudio de los Eunicida, principalmente por su detallada sección sobre la sistemática del orden Eunicida, donde resaltó la importancia de la arquitectura del aparato maxilar para definir los taxa y aclarar sus relaciones filogenéticas. Algunas de sus resultados más interesantes los podemos ver en la delimitación de algunas de las familias, como por ejemplo la inclusión de Lysaretidae en Lumbrineridae, o la de Arabellidae en Oenonidae, así como en la restricción de Dorvilleidae y la propuesta de mantener Iphitimidae 
incluyendo Ophryotrocha y además, propuso 3 de sus nuevos géneros en esta última. Además, formuló una hipótesis de las relaciones filogenéticas entre las familias considerando a las fósiles, en la cual el grado de desarrollo de los aparatos maxilares fue determinante para el agrupamiento de las familias. En su análisis mostró la estrecha relación entre Eunicidae y Onuphidae, con Hartmaniellidae como su grupo hermano; Oenonidae y Lumbrineridae, y un tercer grupo formado por Dorvilleidae, Iphitimidae y Dinophilidae, grupo que posteriormente fue considerado, con base en un estudio filogenético morfológico, como una sola familia: Dorvilleidae (EibyeJacobsen \& Kristensen 1994).

Debe mencionarse que estudios recientes con base en análisis filogenéticos de datos morfológicos y/o moleculares han mostrado que varias de las ideas propuestas por 'Lobo' eran correctas, como la consideración de incluir Lysarete en los Lumbrineridae, la propuesta del género Abyssoninoe, y el restablecimiento de Eranno como un género válido dentro de esta misma familia (Carrera-Parra 2006), o su propuesta de la estrecha relación entre Eunicidae-Onuphidae, así como mantener Dorvilleidae, Iphitimidae y Dinophilidae como familias independientes (Struck et al. 2002, Hall et al. 2004, Struck et al. 2005, Hoisaeter \& Samuelsen 2006, Struck et al. 2006; Rousset et al. 2007). Sin embargo, la inclusión de Dinophilidae dentro de Eunicida no ha sido respaldada en varios estudios (Struck et al. 2002, Hall et al. 2004, Struck et al. 2005, Rousset et al. 2007).

Posteriormente vendrían algunas publicaciones sobre taxonomía con otros autores y, en la mayoría de los casos, dichos trabajos fueron basados en los avances que 'Lobo' había desarrollado y remitido sin reservas. Debe mencionarse que era frecuente su resistencia o negativa a ser coautor de dichos trabajos, pero por la calidad de sus observaciones y dibujos, realmente no había otra opción sino incorporarlo a la lista de autores.

Recorriendo a pie el intermareal de las provincias de Buenos Aires y Chubut en Argentina, de Uruguay o embarcado, 'Lobo' fue un hábil y persistente recolector de material (poliquetos, por supuesto) para estudiarlos en el laboratorio o cederlo generosamente a otros especialistas. Sólo en la Colección Nacional de Invertebrados del Museo Argentino de Ciencias Naturales (MACN-In) depositó 225 lotes con casi 1000 ejemplares. Además, dejó el valor agregado de haber identificado 500 lotes de poliquetos de la colección MACN-In, que incluyen 23 lotes de series tipo de 11 de sus especies.
En síntesis, para 16 familias de poliquetos, 'Lobo' propuso 9 géneros, y describió 27 especies más una subespecie (Anexo 2). Las únicas modificaciones realizadas a dichos taxa radican en que uno de sus géneros, propuesto en la familia Onuphidae (Americonuphis Orensanz, 1974c) devino homónimo secundario de otro propuesto apenas unos meses antes (Americonuphis Fauchald, 1973); esto se explica porque el acceso a la información entonces no era lo que es ahora. El homónimo menor fue reemplazado unos pocos años después. Por otro lado, la única subespecie que propuso (Nothria conchylega anoculata Orensanz, 1974c) fue elevada a especie por Fauchald (1982) y su estatus ha sido confirmado recientemente (Budaeva \& Paxton 2013).

Por otro lado, hace 40 años el 'Lobo' diseñó y llevó al cabo un muestreo intermareal en la Patagonia en dos lugares: uno, frente al entonces Centro Patagónico en Puerto Madryn y en la localidad de San Román (un área protegida en Península Valdés). El muestreo de Puerto Madryn constituyó el tema de tesis de Maestría de Mona (Escofet 1983). En ocasión de la defensa de tesis de M. L. Jaubet, donde 'Lobo' fue jurado, ofreció los datos originales y propuso volver a efectuar el muestreo y comparar ambos sitios 40 años después. Dicho muestreo se realizó en noviembre de 2014 y fue uno de los últimos en los que Lobo participó, aunque a pesar de su condición de cardíaco, nunca dejó de participar en estas actividades. En la playa de Puerto Madryn, 'Lobo' señaló que 14 especies seguían como indescritas. Posiblemente una ya lo fue (Elías et al. 2003), pero otras 13 permanecen sin describir. En el muestreo mencionado, 'Lobo' manifestó su deseo de describir estas especies. Elías y sus colaboradoras se han comprometido a publicar estos resultados.

'Lobo' tuvo dos grandes proyectos de compilación. Uno trató sobre las más de 500 especies de poliquetos bénticos de British Columbia y Washington (Orensanz 1995). El otro es el catálogo de las más de 609 especies de 61 familias repartidas en 2 regiones biogeográficas; esta obra será una referencia importantísima. Ante la insistencia de divulgarlo ampliamente, 'Lobo' había sido ayudado por tres jóvenes colegas y aunque está casi terminado, todavía no está disponible (Orensanz et al. ms). Debe mencionarse que la región que consideraron va de los $30^{\circ} \mathrm{S}$ hasta el extremo sur del continente, más el sector adyacente del Pacífico Sudoriental (hasta los $47^{\circ} \mathrm{N}$ ), y desde la costa y los estuarios hasta los fondos de $2000 \mathrm{~m}$. Este catálogo de registros iba a ser soporte para una base de datos georeferenciada y esperamos que pueda terminarse y hacerse disponible pronto. 


\section{RECONOCIMIENTO INTERNACIONAL}

Para tratar de definir cómo encarar los problemas que enfrentaría en el estudio de los poliquetos, 'Lobo' escribió a los principales especialistas en ese entonces, mismos que hicieron propuestas de ayuda así como recomendaciones relevantes y motivacionales. Edmundo Nonato, recientemente fallecido, le recomendó el 8 de agosto de 1967 que: 'El trabajo más importante, más urgente y quizá más difícil es un estudio de la fauna del Atlántico sudamericano'. Olga Hartman, en carta del 19 de febrero de 1968, comentó: 'Es bueno enterarme de su interés en estudiar los poliquetos de Argentina. Hallará un área casi desconocida que debe ser un reto verdadero'. Menos festivo y más crítico, Kristian Fauchald le comentó el 6 de junio de 1973: 'No me gusta la sinonimia que hizo en Aphrodita, y probablemente estaré en desacuerdo con usted en el futuro, pero sus artículos parecen sólidos'.

El prestigio ganado por 'Lobo' por su quehacer como taxónomo, de la mano de su gran generosidad para compartir sus ideas, materiales y avances concretos, motivó un amplio reconocimiento internacional. En efecto, como homenaje para 'Lobo' se propusieron un nuevo género y 18 especies fueron descritas (Anexo 3), como resultado del trabajo de 27 autores de 8 países: Alemania, Argentina, Chile, España, Estados Unidos de América, México, Rusia y Suecia. Hay una serie de materiales remitidos a otros colegas con lo que consideramos que este tipo de homenaje seguirá todavía por algunos años más. 'Lobo' lo merece y no lo podrá disfrutar porque se nos adelantó el 5 de enero de 2015. Descanse en paz. ¡Hasta siempre, Lobo!

\section{Agradecimientos}

Esta contribución fue enriquecida por los amables comentarios de Ana Parma, Anamaría Escofett y Berenice Trovant. Esperamos que el resultado esté a la altura de sus expectativas.

\section{LiTERATURA CITADA}

Amor A. 1964. El género Dendrostomum (Sipunculida) en la Argentina. Physis 24: 457-470.

Carrera-Parra LF. 2006. Phylogenetic analysis of Lumbrineridae Schmarda, 1861 (Annelida: Polychaeta). Zootaxa 1332: 1-36.

Caso ME. 1964. La labor de don Enrique Rioja como investigador, maestro y amigo de México. Revista de la Sociedad Mexicana de Historia Natural 25: 77-96.
Eibye-Jacobsen D \& RM Kristensen. 1994. A new genus and species of Dorvilleidae (Annelida, Polychaeta) from Bermuda, with a phylogenetic analysis of Dorvilleidae, Iphitimidae and Dinophilidae. Zoological Scripta 23: 107131.

Escofet A. 1983. Community ecology of a sandy beach from Patagonia (Argentina, South America). Thesis, Master of Science, University of Washington, Seattle, $122 \mathrm{pp}$.

Hall KA, PA Hutchings \& DJ Colgan. 2004. Further phylogenetic studies of the Polychaeta using 18S rDNA sequence data. Journal of the Marine Biological Association of the United Kingdom 84: 949-960.

Hoisaeter T \& TJ Samuelsen. 2006. Taxonomic and biological notes on a species of Iphitime (Polychaeta, Eunicida) associated with Pagurus prideaux from western Norway. Marine Biology Research 2: 333-354.

Olivier SR. 1962. Los cladóceros argentinos, claves de las especies, notas biológicas y distribución geográfica. Revista del Museo de La Plata (Nueva Serie), Zoología 7: 173-269.

Orensanz J. 2010. Historias de mi pueblo: Santa Clara del Mar 1: 1-212. Secretaría de Cultura y Educación del Partido de Mar Chiquita, Santa Clara del Mar.

Orensanz JM. 1990. The eunicemorph polychaetes from Antarctic and Subantarctic Seas. Antarctic Research Series 52: $1-183$.

Orensanz JM. 1995. Benthic polychaetes of British Columbia and Washington, 43 pp. Evolving Update, Seattle.

Orensanz JM. 2009. Prólogo. In: de León-González JA, JR Bastida-Zavala, LF Carrera-Parra, ME García-Garza, A Peña-Rivera, SI Salazar-Vallejo \& V Solís-Weiss (eds). Poliquetos (Annelida: Polychaeta) de México y América Tropical, p. 6, Universidad Autónoma de Nuevo León, Monterrey.<http://www.fcb.uanl.mx/silpoly/index.html>.

Rousset V, F Pleijel, G Rouse, C Erséus \& M Siddall. 2007. A molecular phylogeny of annelids. Cladistics 23: 41-63.

Salazar-Vallejo SI, P Gillet \& LF Carrera-Parra. 2007. Revision of Chauvinelia, redescriptions of Flabelliseta incrusta, and Helmetophorus rankini, and their recognition as acrocirrids (Polychaeta: Acrocirridae). Journal of the Marine Biological Association of the United Kingdom 87: 465-477.

Struck TH, G Purschke \& K Halanych. 2006. Phylogeny of Eunicida (Annelida) and exploring data congruence using a Partition Addition Bootstrap Alteration (PABA) Approach. Systematic Biology 55: 1-20.

Struck TH, KM Halanych \& G Purschke. 2005. Dinophilidae (Annelida) is most likely not a progenetic Eunicida: Evidence from 18S and 28S rDNA. Molecular Phylogenetics and Evolution 37: 619-623. 
Struck TH, W Westheide \& G Purschke. 2002. Progenesis in Eunicida («Polychaeta,» Annelida) -Separate evolutionary events? Evidence from molecular data. Molecular Phylogenetics and Evolution 25: 190-199.
Wolf M. 2009. Galina Tolmacheva: El actor en el centro del acontecimiento. In: Dubatti J (coord.). Historia del Actor 2: Del ritual Dionisíaco a Tadeusz Kantor, pp 325-357 Colihue, Buenos Aires.

Recibido el 6 de abril de 2015 y aceptado el 26 de agosto de 2015

Editor: Claudia Bustos D. 
Anexo 1. Lista de las publicaciones realizadas por Lobo en ecología del bentos y sobre poliquetos (PDFs disponibles con Ana Parma, Luis 0 Sergio) / List of Lobo publications in benthic ecology or polychaetes (PDFs available with Ana Parma, Luis or Sergio)

Barnich R, JM Orensanz \& D Fiege. 2012. Remarks on some scale worms (Polychaeta, Polynoidae) from the Southwest Atlantic with notes on the genus Eucranta Malmgren, 1866 and description of a new Harmothoe species. Marine Biodiversity 42: 395-410

Böggemann M \& JM Orensanz. 2007. Glyceriformia Fauchald, 1977 (Annelida: 'Polychaeta') from the SW Atlantic Shelf, between $30^{\circ}$ and $45^{\circ} \mathrm{S}$. Mitteilungen aus dem Hamburgischen Zoologischen Museum und Institut 104: 11-59.

Carrera-Parra L \& JM Orensanz. 2002. A revision of the genus Kuwaita (Polychaeta, Lumbrineridae). Zoosystema 24: 273-281.

Diez ME, JM Orensanz, F Márquez \& F Cremonte. 2013. Shell damage in the Tehuelche scallop Aequipecten tehuelchus caused by Polydora rickettsi (Polychaeta: Spionidae) infestation. Journal of Invertebrate Pathology 114: 107-113.

Diez ME, VI Radashevsky, JM Orensanz \& F Cremonte. 2011. Spionid polychaetes (Annelida: Spionidae) boring into shells of molluscs of commercial interest in northern Patagonia, Argentina. Italian Journal of Zoology 78: 497504.

Elías R \& JM Orensanz. 2014. Poliquetos. In: Calcagno J (ed). Los invertebrados marinos, 354 pp. Fundación de Historia Natural Félix de Azara, Buenos Aires.

Elías R, CS Bremec, PC Lana \& JM Orensanz. 2003. Opheliidae (Polychaeta) from the southwestern Atlantic Ocean, with the description of Travisia amadoi n. sp., Ophelina gaucha n. sp. and Ophelina alata n. sp. Hydrobiologia 496: 75-85.

Escofet A, JM Orensanz, SR Olivier \& V Scarabino. 1978. Biocenología béntica del golfo San Matías (Río Negro, Argentina). Anales del Centro de Ciencias del Mar y Limnología, UNAM 5: 59-82.

Obenat S, E Spivak \& JM Orensanz. 2006. Reproductive biology of the invasive reef-forming serpulid Ficopomatus enigmaticus (Serpulidae) in the Mar Chiquita Coastal Lagoon, Argentina. Journal of Invertebrate Reproduction 49: 263-272.

Olivier SR, A Escofet, PE Penchaszadeh \& JM Orensanz. 1972a. Estudios ecológicos en la región estuarial de la albufera de Mar Chiquita, 1. Las comunidades bentónicas. Anales de la Sociedad Científica Argentina 193: 237-262.

Olivier SR, A Escofet, PE Penchaszadeh \& JM Orensanz. 1972b. Estudios ecológicos en la región estuarial de la albufera de Mar Chiquita, 2. Las relaciones tróficas interespecíficas. Anales de la Sociedad Científica Argentina 194: 89-104.
Olivier SR, A Escofet, JM Orensanz, SE Pezzani, AM Turró \& ME Turró. 1966. Contribución al conocimiento de las comunidades bentónicas de Mar del Plata, 1. Las costas rocosas entre Playa Grande y Playa Chica. Anales de la Comisión de Investigación Científica de la Provincia de Buenos Aires (Argentina) 7: 185-206.

Orensanz JM. 1973a. Los anélidos poliquetos de la provincia biogeográfica argentina, 1. Palmyridae, Amphinomidae y Euphrosinidae. Physis 83: 485-502.

Orensanz JM. 1973b. Los anélidos poliquetos de la provincia biogeográfica argentina, 2. Aphroditidae. Physis 83: 503518.

Orensanz JM. 1974a. Los anélidos poliquetos de la provincia biogeográfica argentina, 3. Dorvilleidae. Physis 85: 325 342 .

Orensanz JM. 1974b. Los anélidos poliquetos de la provincia biogeográfica argentina, 4. Lumbrineridae. Physis 85: 343 393.

Orensanz JM. 1974c. Los anélidos poliquetos de la provincia biogeográfica argentina, 5. Onuphidae. Physis A 33(86): 75-122.

Orensanz JM. 1974d. Los anélidos poliquetos de la provincia biogeográfica argentina, 6. Arabellidae. Physis A 33(87): 381-408.

Orensanz JM. 1975a. Los anélidos poliquetos de la provincia biogeográfica argentina, 7. Eunicidae y Lysaretidae. Physis A 34(88): 85-111.

Orensanz JM. 1975b. Los anélidos poliquetos de la provincia biogeográfica argentina, 8. Polygordidae. Neotropica 20(62): $87-90$.

Orensanz JM. 1975c. Los anélidos poliquetos de la provincia biogeográfica argentina, 10. Acrocirridae. Neotropica 20 (63): 113-118.

Orensanz JM. 1976a. Los anélidos poliquetos de la provincia biogeográfica argentina, 9. Poecilochaetidae y Cossuridae. Comunicaciones Zoológicas del Museo de Historia Natural de Montevideo 10 (140): 1-8.

Orensanz JM. 1976b. Los anélidos poliquetos de la provincia biogeográfica magallánica, 1. Catálogo de las especies citadas hasta 1974. Laboratorio de Comunidades Bentónicas, Contribución Técnica 1: 1-83.

Orensanz JM. 1977. Polychaeta. In: Hurlbert SH (ed). Biota acuática de Sudamérica Austral, pp. 97-98. San Diego State University, San Diego.

Orensanz JM. 1981. Polychaeta. In: Hurlbert SH, G Rodriguez \& ND Santos (eds). Aquatic biota of Tropical South America, Part 2: Anarthropoda, pp. 167-169. San Diego State University, San Diego. 
Orensanz JM. 1982. Polychaeta. In: Hurlbert SH \& A Villalobos-Figueroa (eds). Aquatic biota of Mexico, Central America and the West Indies, pp 159-161. San Diego State University, San Diego.

Orensanz JM. 1990. The eunicemorph polychaetes from Antarctic and Subantarctic Seas. Antarctic Research Series 52: $1-183$.

Orensanz JM \& MC Estivariz. 1972. Los anélidos poliquetos de aguas salobres de la provincia de Buenos Aires, Argentina. Revista del Museo de La Plata (Argentina) 11(Zoología 98): 95-104.

Orensanz JM \& VF Gallucci. 1982. Post-catastrophic recovery in the marine benthos: process and management implications. Monografías Biológicas 2: 181-198, Pontificia Universidad Católica de Chile, Santiago.

Orensanz JM \& NM Gianuca. 1974. Contribuiçao ao conhecimento dos anelideos poliquetos do Rio Grande do Sul, Brasil, 1. Lista sistematica preliminar e descricao de tres novas especies. Comunicaçoes Zoológicas do Museu de Ciencias da Pontificia Universidade Católica de Rio Grande do Sul (Porto Alegre, Brasil) 4: 1-37.

Orensanz JM \& F Ramírez. 1973. Taxonomía y distribución de los poliquetos pelágicos del Atlántico Sudoccidental. Boletín del Instituto de Biología Marina, Mar del Plata, Argentina 21: 1-86.

Orensanz JM, F Ramírez \& EO Dinofrio. 1974. Resultados planctológicos de la Campaña 'OCEANTAR I', 2. Poliquetos. Contribuciones del Instituto Antártico Argentino 184: $1-41$.
Orensanz JM, E Schwindt, G Pastorino, A Bortolus, G Casas, G Darrigran, R Elías, JJ López-Gappa, S Obenat, M Pascual, P Penchaszadeh, ML Piriz, F Scarabino, ED Spivak \& E Vallarino. 2002. No longer the pristine confines of the world ocean- A survey of exotic marine species in the Southwestern Atlantic. Biological Invasions 4: 115-143.

Orensanz JM, E Bogazii \& AM Parma. 2008. Impacto de la pesca sobre el subsistema bentónico En: Estado de Conservación del Mar Patagónico, pp. 678-698. AquaMarina y otras 9 organizaciones, Buenos Aires. <http:/ /www.marpatagonico.org/libro/articulo.php?id=orensanzbogazzi-parma-impacto-subsistema-bentonico>

Salazar-Vallejo SI \& JM Orensanz. 1991. Pilárgidos (Annelida: Polychaeta) de Uruguay y Argentina. Cahiers de Biologie Marine 32: 267-279.

Salazar-Vallejo SI \& JM Orensanz. 2007. Pleijelius longae n. gen., n. sp., a remarkable deep water polychaete from the Northwestern Atlantic (Polychaeta: Hesionidae). In: Sardá R, G San Martín, E López, D Martín \& D George (eds). Scientific advances in polychaete research. Scientia Marina 70(Supl. 3): 157-166.

Schwindt E, J López-Gappa, MP Raffo, M Tatián, A Bortolus, JM Orensanz, G Alonso, ME Diez, B Doti, G Genzano, C Lagger, G Lovrich, ML Piriz, MM Méndez, V Savoya \& MC Sueiro. 2014. Marine fouling invasions in ports of Patagonia (Argentina) with implications for legislation and monitoring programs. Marine Environmental Research 99: 60-68. 
Anexo 2. Lista sistemática de los nuevos taxa propuestos o descritos por Lobo entre los poliquetos; las familias de poliquetos y los taxa en orden alfabético. En algunos casos se agregan comentarios nomenclaturales y las referencias de los mismos / Systematic list of the new taxa proposed or described by Lobo in polychaetes; families and taxa arranged in alphabetic order. In some cases, nomenclatural comments and their references are included

\section{TAXA}

Acrocirridae

Flabelligella erratica Orensanz, 1975c

Chrysopetalidae

Cossuridae

Paleanotus intermedius Orensanz, 1973a

Cossura heterochaeta Orensanz, 1976a

Dorvilleidae

Pettiboneia Orensanz, 1974a (enmendado por Blake, 1979)

Pettiboneia hartmanae Orensanz, 1990

Pettiboneia sanmatiensis Orensanz, 1974a [aparentemente redescrita por Blake, 1979; en realidad los ejemplares de California y British Columbia difieren en varios aspectos como la posición de los ocelos (posteriores a las antenas $v s$ anteriores en el material tipo), tipo de furcadas (ramas de distinta longitud, la más corta con crestas basales transversas vs de la misma longitud y lisas en tipo), y forma de las mandíbulas (delgadas con dientes redondeados $v s$ Eunicidae gruesas con dientes escasamente proyectados)].

Eunice austropacifica Orensanz, 1990

Marphysa galluccii Orensanz, 1990

Glyceridae

Hemipodus olivieri Orensanz \& Gianuca, 1974 [considerada como sinónimo menor de $H$. californiensis (Hartman, 1938) por Böggemann (2002), pero no lo son. En H. olivieri el lóbulo presetal tiende a reducirse hacia la región posterior, mientras que en $H$. californiensis sigue marcadamente proyectado en setígeros posteriores].

Goniadidae

Glycinde henningi Böggemann \& Orensanz, 2007

Hesionidae

Pleijelius Salazar-Vallejo \& Orensanz, 2007

Pleijelius longae Salazar-Vallejo \& Orensanz, 2007 Iphitimidae

Mammiphitime Orensanz, 1990

Mammiphitime tridentata Orensanz, 1990

Palpiphitime Orensanz, 1990

Pinniphitime Orensanz, 1990

Pinniphitime pinnognatha Orensanz, 1990

Lumbrineridae

Abyssoninoe Orensanz, 1990

Lumbrinerides Orensanz, 1974b

Lumbrinerides gesae Orensanz, 1974b

Lumbrineriopsis Orensanz, 1974b

Lumbrineris knipovichana Orensanz, 1974b

Oenonidae (Arabellidae)

Arabella (Arabella) protomutans Orensanz, 1990

Drilonereis simplex Orensanz, 1974

Drilonereis viborita Orensanz, 1990

Onuphidae

Americonuphis Orensanz, 1974c (homónimo secundario de Americonuphis Fauchald, 1973; reemplazado por Australonuphis Paxton, 1979)

Americonuphis casamiquelorum Orensanz, 1974c (= Australonuphis casamiquelorum (Orensanz, 1974c).

Hyalinoecia incubans Orensanz, 1990

Leptoecia vivpara Orensanz, 1990

Nothria conchylega anoculata Orensanz, 1974c (elevada a especie como Nothria anoculata Orensanz, 1974c por Fauchald, 1982; confirmado por Budaeva \& Paxton 2013)

Onuphis oligobranchiata Orensanz, 1974c (= Kinbergonuphis oligobranchiata (Orensanz, 1974c) fide Fauchald, 1982).

Opheliidae

Ophelina alata Elías, Bremec, Lana \& Orensanz, 2003

Ophelina gaucha Elías, Bremec, Lana \& Orensanz, 2003

Travisia amadoi Elías, Bremec, Lana \& Orensanz, 2003

Polynoidae

Harmothoe madrynensis Barnich, Orensanz \& Fiege, 2012

Sigalionidae

Sigalion cirrifer Orensanz \& Gianuca, 1974 Spionidae

Spio (Microspio) gaucha Orensanz \& Gianuca, 1974 


\section{LITERATURA CITADA}

Blake JA. 1979. A redescription of Pettiboneia sanmatiensis Orensanz (Polychaeta: Dorvilleidae) and a revised key to the genera of Dorvilleidae. Bulletin of the Southern California Academy of Sciences 78: 136-140.

Böggemann M. 2002. Revision of the Glyceridae Grube 1850 (Annelida, Polychaeta). Abhandlungen der Senckenbergischen Naturforschenden Gesellschaft Frankfurt am Main 555: 1-249.

Budaeva N \& H Paxton. 2013. Nothria and Anchinothria (Annelida: Onuphidae) from eastern Australian waters, with a discussion of ontogenetic variation of diagnostic characters. Journal of the Marine Biological Association of the United Kingdom 93: 1481-1502.
Fauchald K. 1973. Polychaetes from Central American sandy beaches. Bulletin of the Southern California Academy of Sciences 72: 19-31.

Fauchald K. 1982. Revision of Onuphis, Nothria, and Paradiopatra (Polychaeta: Onuphidae) based upon type material. Smithsonian Contributions to Zoology 356: 1109.

Paxton H. 1979. Taxonomy and aspects of the life history of Australian beachworms (Polychaeta: Onuphidae). Australian Journal of Marine and Freshwater Research 30: 26-294.

Anexo 3. Lista alfabética y referencias de los taxa nombrados en honor de Lobo Orensanz / Alphabetic list and references for taxa named after Lobo Orensanz

\section{TAXA}

\section{Género}

Loboneris Carrera-Parra, 2006 (Polychaeta, Lumbrineridae).

\section{Especies}

Abyssoninoe orensanzi Hartmann-Schröder, 1992

(Polychaeta, Lumbrineridae).

Armandia loboi Elías \& Bremec, 2003 (Polychaeta,

Opheliidae).

Eunice orensanzi de León-González, 1992

(Polychaeta, Eunicidae).

Flabelliigera orensanzi Salazar-Vallejo, 2012

(Polychaeta, Flabelligeridae).

Lamelleulepethus orensanzi Pettibone, 1986 (Polychaeta, Eulepethidae).

Haplosyllis loboi Paola, San Martín \& Martin, 2006 (Polychaeta, Syllidae).

Lumbrineris orensanzi Hartmann-Schroder, 1980 (Polychaeta, Lumbrineridae).

Marphysa orensanzi Carrera-Parra \&, SalazarVallejo, 1998 (Polychaeta, Eunicidae).

Nicon orensanzi de León-González \& Trovant, 2013 (Polychaeta, Nereididae).

Noanelia orensanzi Schüller \& Jirkov, 2013 (Polychaeta, Ampharetidae).

Onuphis orensanzi Fauchald, 1982 (Polychaeta, Onuphidae).

Ophryotrocha orensanzi Taboada, Wiklund, Glover, Dahlgren, Cristobo \& Avila, 2013

(Polychaeta, Dorvilleidae).
Paratherochaeta orensanzi Salazar-Vallejo, 2013 (Polychaeta, Flabellgeridae)

Prionospio orensanzi Blake, 1983 (Polychaeta, Spionidae).

Protodorvillea orensanzi Carrasco \& Palma, 2000

(Polychaeta, Dorvilleidae).

Puelche orensanzi Barnard \& Clark, 1982

(Amphipoda, Phoxocephalopsidae).

Sabellaria orensanzi Kirtley, 1994 (Polychaeta, Sabellariidae).

Trophoniella orensanzi Salazar-Vallejo, 2012 (Polychaeta, Flabelligeridae).

\section{LITERATURA CITADA}

Barnard JL \& J Clark. 1982. Puelche orensanzi, new genus, new species, a phoxocephalopsid amphipod from the shores of Argentina (Crustacea: Amphipoda: Phoxocephalopsidae). Journal of Crustacean Biology 2: 261272.

Blake JA. 1983. Polychaetes of the family Spionidae from South America, Antarctica, and adjacent seas and islands. Antarctic Research Series 39: 205-288.

Carrasco FD \& M Palma. 2000. Two new species of Pettiboneia and Protodorvillea (Dorvilleidae, Polychata) from Northern Chile. Bulletin of Marine Science 67: $597-$ 602.

Carrera-Parra LF. 2006. Revision of Lumbrineris de Blainville, 1828 (Polychaeta: Lumbrineridae). Zootaxa 1336: 1-64. 
Carrera-Parra LF \& SI Salazar-Vallejo. 1998. A new genus and 12 new species of Eunicidae (Polychaeta) from the Caribbean Sea. Journal of the Marine Biological Association of the United Kingdom 78: 1-36.

De León-González JA \& B Trovant. 2013. A new species of Nicon Kinberg, 1866 (Polychaeta, Nereididae) from Ecuador, Eastern Pacific, with a key to all known species of the genus. ZooKeys 269: 67-76.

Elías R \& CS Bremec. 2003. First record of the genus Armandia (Opheliidae, Polychaeta) in Argentine waters, with the description of Armandia loboi sp. n. Bulletin of Marine Science 72: 181-186.

Fauchald K. 1982. Revision of Onuphis, Nothria, and Paradiopatra (Polychaeta: Onuphidae) based upon type material. Smithsonian Contributions to Zoology 356: 1109.

Hartmann-Schröder G. 1980. Die Polychaeten der tropischen Nordwestküste Australiens (zwischen Port Samson im Norden und Exmouth im Süden). Mitteilungen aus dem Hamburgischen Zoologischen Museum und Institut 77: 41110 .

Kirtley DW. 1994. A review and taxonomic revision of the family Sabellariidae Johnston 1865 (Annelida; Polychaeta). Sabecon Press, Vero Beach, Science Series 1: 1-223.
Paola A, G San Martín \& D Martin. 2006. A new species of Haplosyllis Langerhans, 1879 (Annelida: Polychaeta: Syllidae: Syllinae) from Argentina. Proceedings of the Biological Society of Washington 119: 346-354.

Pettibone MH. 1986. Additions to the family Eulepethidae Chamberlin (Polychaeta: Aphroditacea). Smithsonian Contributions to Zoology 441: 1-51.

Salazar-Vallejo SI. 2012. Revision of Flabelligera Sars, 1829 (Polychaeta: Flabelligeridae). Zootaxa 3203: 1-64.

Salazar-Vallejo SI. 2012. Revision of Trophoniella Hartman, 1959 (Polychaeta, Flabelligeridae). Zoosystema 34: 453519.

Salazar-Vallejo SI. 2013. Revision of Therochaeta Chamberlin, 1919 (Polychaeta: Flabelligeridae). Zoosystema 35: 95-131

Schüller M \& IA Jirkov. 2013. New Ampharetidae (Polychaeta) from the deep Southern Ocean and shallow Patagonian waters. Zootaxa 3692: 204-237.

Taboada S, H Wiklund, AG Glover, TG Dahlgren, J Cristobo \& C Avila. 2013. Two new Antarctic Ophryotrocha (Annelida: Dorvilleidae) described from shallow-water whale bones. Polar Biology 36: 1031-1045. 